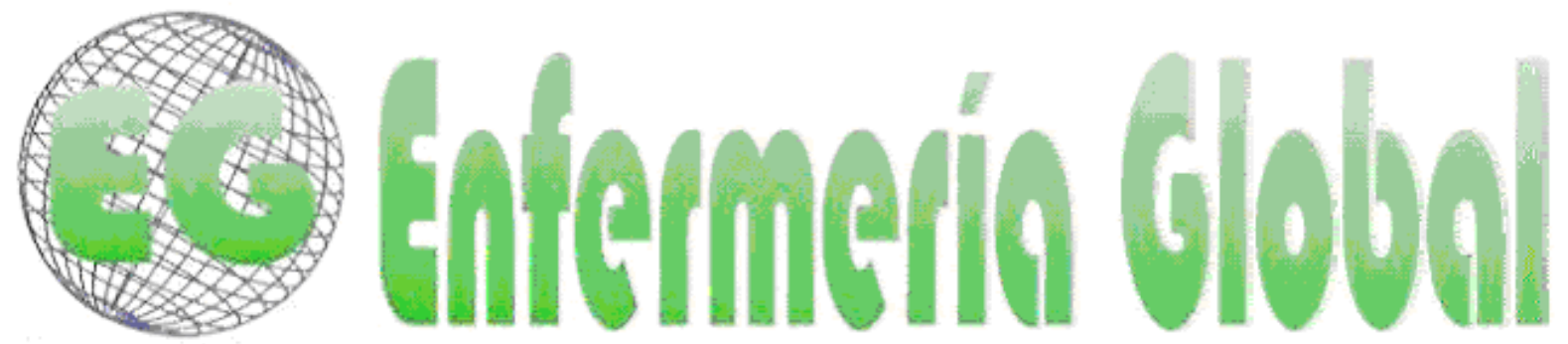

\title{
Hostigamiento laboral: amenaza permanente para enfermería
}

Harassment in the workplace: A permanent threat for nursing

\section{*Dois Castellón, Angelina María}

\author{
*Enfermera Matrona, Magíster en Psicología de la Salud, Profesora Asociada, Escuela de Enfermería \\ Pontificia Universidad Católica de Chile. E-mail: adois@uc.cl
}

Palabras clave: hostigamiento laboral; enfermería; factores involucrados.

Keywords: harassment in the workplace; nursing; involved factors.

\section{RESUMEN}

El hostigamiento laboral es definido como una situación donde una persona es, repetidamente en el tiempo, objeto de actos negativos en el trabajo y de los cuales no puede defenderse, siendo tan graves sus consecuencias potenciales que se lo ha catalogado como una de las formas de estrés social más severo que se puede enfrentar.

La evidencia internacional sugiere que la cuarta parte de la violencia laboral se dirige contra trabajadores del sector salud y en especial contra las enfermeras. En Chile existe escasa evidencia que dé cuenta de la realidad del hostigamiento laboral en enfermería, razón por la cual el objetivo de esta investigación es describir, usando metodología cualitativa, los factores involucrados en la ocurrencia y mantención de este fenómeno a partir de las experiencias de enfermeras que han sido víctimas de él.

Los principales resultados muestran que tanto elementos organizacionales como relativos a la ejecución del rol profesional actuarían como agentes favorecedores del hostigamiento laboral e influirían directamente en el desarrollo del ciclo de violencia. Por otro lado, las formas en que las enfermeras describen los hechos, las consecuencias en las mismas y las reacciones de los testigos influirían en la mantención del hostigamiento en el tiempo.

\section{ABSTRACT}

Harassment in the workplace is defined as a situation where a person is repeatedly the object of negative acts in the workplace from which he/she cannot defend, with potential consequences being so serious to him/her that they have been catalogued as one of the most severe forms of social stress that it is possible to face.

International evidence suggests that a quarter of violence in the workplace is directed towards health workers, especially against nurses. In Chile, there is little evidence to account for the harassment of 
nurses. For that reason the aim of this research is to describe using qualitative methodology the factors involved in the occurrence this phenomenon and support for the nurses who have been victims of it.

The results show that the factors that directly influence the development of the cycle of violence are the characteristics of the organization and the form of development in the professional role. On the other hand, the ways in which the nurses describe the facts, the consequences and the reactions of the witnesses would prolong the harassment.

\section{INTRODUCCIÓN}

El hostigamiento laboral en enfermería ha sido definido por el Consejo Internacional de Enfermeras, junto a la Organización Mundial de la Salud, como incidentes donde el equipo es víctima de abuso, amenazas o agresión en circunstancias relacionadas con el trabajo y que involucra cambios implícitos o explícitos en la seguridad, bienestar o salud de los afectados ${ }^{(1)}$.

La evidencia internacional disponible sugiere que la cuarta parte de la violencia en el lugar de trabajo se dirige contra trabajadores del sector salud y de ellos, las enfermeras serían las más susceptibles a experimentarlo ${ }^{(2,3)}$.

Diferentes estudios internacionales han mostrado una prevalencia de hostigamiento laboral en enfermeras que varia entre un $10 \%$ y un $50 \%$, llegando incluso a reportarse índices del $87 \%^{(4,5)}$.

Los estudios dan cuenta de que el hostigamiento se asocia a alteraciones físicas y mentales de las víctimas y a niveles elevados de licencias médicas. Además, las enfermeras reconocen en su mayoría a sus jefes directos como responsables de las conductas hostigantes y a un tercio de sus colegas como sus principales agresoras ${ }^{(5,6)}$

En Chile existe escasa evidencia que dé cuenta del alcance de este fenómeno en enfermería, razón por la cual el objetivo de esta investigación es describir los factores involucrados en la ocurrencia y mantención del hostigamiento laboral a partir de las experiencias de enfermeras que han sido víctimas de él.

\section{MATERIAL Y MÉTODO}

Estudio cualitativo descriptivo que utilizó como guía la teoría fundada en los datos -Grounded Theory- de Glaser y Strauss ${ }^{(7,8)}$

\section{Participantes}

Para la selección de las participantes y por la dificultad de acceso a ellas, se utilizó muestreo por conveniencia y en cadena intentando respetar al máximo los criterios de pertinencia, adecuación, conveniencia, oportunidad y disponibilidad para la obtención de los datos ${ }^{(9)}$. Los Criterios de Inclusión consideraron a enfermeras y/o enfermerasmatronas chilenas, dispuestas a firmar consentimiento informado, que se identificaran a si mismas como víctimas de hostigamiento laboral durante el ejercicio de la profesión en instituciones de salud públicas o privadas, excluyendo situaciones de violencia física y/o acoso sexual y violencia de cualquier tipo ejercida por usuarios del sistema de salud. 


\section{Procedimiento}

Para la recolección de los datos se usó la entrevista semi-estructurada a partir de un guión temático, previamente validado y modificado con tres entrevistas pilotos a enfermeras que cumplían con los criterios de inclusión. Cada entrevista fue grabada, transcrita y analizada en su totalidad dando inicio al proceso de codificación abierta. Una vez terminada esta primera codificación se procedió a realizar la segunda entrevista y, siguiendo el mismo procedimiento, se procedió a codificarla. A la luz de dichas codificaciones se hicieron modificaciones en el guión de entrevista para profundizar en algunos temas, procedimiento que se repitió a partir del análisis de las siguientes entrevistas.

Se realizaron trece entrevistas, entre enero y mayo de 2007. El criterio de finalización fue la saturación temática ${ }^{(10)}$. Los datos se analizaron de acuerdo a los procedimientos de la Grounded Theory ${ }^{(11)}$, utilizando la codificación abierta para identificar los conceptos emergentes, sus propiedades y dimensiones. La rigurosidad metodológica se aseguró aplicando los criterios de Guba ${ }^{(12)}$ de manera que la descripción ofrecida por la investigadora represente fielmente la experiencia de las participantes Una vez finalizado el análisis descriptivo los resultados fueron triangulados con tres expertos en el tema de manera de disminuir el sesgo de investigación.

Las consideraciones éticas de este estudio se originan a partir del resguardo de la dignidad de la persona durante toda investigación, considerando el consentimiento informado aprobado para el Proyecto de Investigación № 0702005010 (Purdue University Institucional Review Board) que asegura la confidencialidad de la información, la participación voluntaria y el respeto por la autonomía de las enfermeras participantes.

\section{RESULTADOS}

La muestra quedó constituida por 13 enfermeras y/o enfermeras-matronas formadas en universidades chilenas tradicionales, que habían vivido experiencias de hostigamiento laboral. Los datos que se presentan corresponden a las características de la muestra al momento de la ocurrencia de los hechos. La edad promedio de las participantes fue de 36.5 años, con un rango de 23 a 56 años. 7 eran solteras, 3 casadas y 3 separadas. Del total, 3 percibía los ingresos que recibía por su trabajo como relevantes para su mantención personal y/o de su familia y a excepción de una de ellas, el resto vivía con sus familias. Del total de enfermeras entrevistadas, 10 trabajaban en servicios hospitalarios (públicos y privados), 2 en centros públicos de Atención Primaria y 1 en docencia universitaria. Del total de enfermeras, 3 se desempeñaba en cargos que requerían del trabajo en turnos, el resto sólo cumplía con jornada diurna. Los años promedio de desempeño en ese cargo fueron de 3.9 años con un rango que varió entre 6 meses y 14 años. La duración promedio del hostigamiento laboral fue de 19 meses con un rango entre 2 meses y 7 años. Entre las personas que ejecutaban conductas de hostigamiento, 11 eran enfermeras y de ellas, 9 cumplían labores de jefaturas. En un caso el hostigador era médico y hombre y en un sólo caso la hostigadora era una auxiliar paramédico. Del total de enfermeras, una de ella había sido víctima de hostigamiento laboral previamente. 
A partir de los datos obtenidos de las entrevistas se identificaron 10 categorías relacionadas directamente con la visión de las enfermeras del fenómeno de hostigamiento laboral:

\section{Descripción de los contextos organizacionales/institucionales}

Las enfermeras describieron algunas características organizacionales que, según ellas, facilitaban o permitían la ocurrencia de hostigamiento laboral en enfermería agrupadas en cuatro factores relacionados con la forma de resolver los conflictos en la institución, con los sistemas de recompensa e incentivo, con el ambiente laboral y con las características de las jefaturas y directivos no directamente involucrados en las conductas de hostigamiento

En relación a los primeros, las enfermeras describieron la ausencia de políticas de recursos humanos que hicieran explícita una metodología organizacional preventiva tendiente a resolver los conflictos a través de las constancias por escrito de los hechos y de los compromisos y los tiempos de resolución de los mismos. Además, describieron prácticas que tendían a invisibilizar la situación de violencia mediante la falta de investigación de los hechos, falta de respuesta a las denuncias y ausencia de sanciones a los perpetradores de actos violentos. Por otro lado, describen que en estas organizaciones los objetivos estaban centrados en la producción y no en los trabajadores:

“... además de ofrecer servicios de salud para el resto, la salud de los propios funcionarios también tiene que estar siendo tomada en cuenta y las necesidades que tengan... yo creo que priorizan otros aspectos, como poca conciencia y cultura de cuidar al funcionario... lo que se busca es que produzca mucho..." (E9, 198)

lo que en algunas ocasiones se traducía en sistemas de recompensa perversos e inamovilidad laboral y en la ausencia de una política clara de ascenso laboral.

En relación a los ambientes de trabajo donde ocurre el hostigamiento laboral, las enfermeras refirieron que éstos se caracterizaban por ser rígidos, autoritarios, jerárquicos y con liderazgos burocráticos:

"... y es como un tipo de liderazgo distinto que funcionaba en base a un tipo de liderazgo como mucho más, más rígido, eh... y más vertical..." $(H 12,14)$.

Asimismo, estos ambientes tienden a ser fríos, impersonales, hostiles, con una alta carga de trabajo y con poco personal para desarrollarlo y en los que no se permitía la expresión explícita de los conflictos:

"... no les gusta que haya conflictos ahí, osea, hay que estar todo solapado y todo escondido y que si hay conflicto se discutan en otro lado...." (E7, 21).

Por último, en relación a las características de las personas que tenían cargos de jefaturas y que no estaban directamente involucradas en los actos de hostigamiento, éstas fueron descritas desde dos posiciones diferentes. Las primeras, con una tendencia a minimizar el conflicto interviniendo de forma tal que éste no se enfrentaba o la intervención no era suficiente: 
“...o sea, como se te ocurre, como minimizando, bajándole el perfil, cuando en el fondo igual ha sido testigo de los que pasa con otros colegas y que es la misma situación..." (E12, 62)

y la segunda, menos frecuente, apoyando a la víctima e interviniendo activamente sobre la situación de hostigamiento:

“...lo raro era que la enfermera jefe no tuviera la misma actitud, siendo ellas super amigas, y yo lo probé muchas veces, ella me ayudó, ella respondió bien, siempre me ayudó..." (H11, 29).

Cabe destacar que, en general, las enfermeras esperaban una intervención activa de parte de las jefaturas y por lo mismo se sintieron defraudadas del accionar de las mismas, lo que se tradujo en una sensación de falta de apoyo institucional frente al conflicto.

\section{Características de las personas que perpetran actos de hostigamiento laboral}

En relación a las personas que actuaron como hostigadoras, destaca que la mayoría eran enfermeras que se desempeñaban como jefas de los servicios donde trabajaba la víctima.

En el ámbito personal, estas personas fueron descritas como envidiosas, suficientes, agresivas, impredecibles, mentirosas, intolerantes, irascibles, ambivalentes, no reconocen ni aceptan sus equivocaciones, ni la supervisión de su trabajo y con problemas de autoestima, lo que las hacia sentirse en una posición vulnerable frente a las víctimas:

“...creo que ella se sintió amenazada en su cargo, eh, creo que ella sintió de cierta forma que entró a competir conmigo... yo creo que se sintió amenazada en algún momento..." (E10, 36).

En relación a las características profesionales, éstas se agrupan en aquellas relacionadas con el trato a las personas y las relacionadas con la necesidad de ostentar y mantener el poder. Según el trato hacia las personas, las enfermeras describieron a sus hostigadores/as como sujetos que generaban temor $y$ desconfianza en el personal que tenían a cargo y que se caracterizaban por un trato castigador y maltratador:

“...y ellas fueron terriblemente abusadoras conmigo y no solamente abusadoras en el trabajo mismo sino que además en castigarte cada vez que podían donde tu estabai..." (E7, 18).

En relación a la necesidad de ostentar y mantener el poder, fueron descritas como personas que necesitaban mantener el control de las situaciones:

"...es que, es que ella era una persona que necesita controlar todo y controlar a la gente como títeres, si tu no eres como títere con ella, tu no puedes seguir con ella, lograr nada" (E13,62),

para lo cual utilizaban estrategias en el plano laboral tales como hacer propios los logros de otras personas, hacer alarde de sus propios logros y mantenerse siempre 
en una posición de apoyo a las jefaturas de mayor rango, por sobre el personal que tenían a cargo, de manera de mantener además el estatus asociado al poder que enviste el cargo:

“... maneja mucho los hilos de todas las personas... sabe las puntadas que tiene que dar para cocer bien... siempre le saca provecho a las situaciones..." $(E 1,43)$

\section{Características de las personas que son víctimas de actos de hostigamiento laboral}

Las enfermeras describieron a las personas que son víctimas de actos de hostigamiento laboral, tanto ellas como otros, desde dos puntos de vista. Uno relacionado con sus características personales y el otro relacionado con la forma de ejecutar su trabajo.

Las características personales fueron agrupadas de dos formas, las primeras como simpáticas, empáticas, de carácter fuerte y confrontacional, estrictas, meticulosas, autoexigentes, estudiosas y directas.

Las segundas fueron descritas como calladas, sumisas:

“... tiene que ver mucho con la personalidad de sumisión, eso de parecer descalza, que el delantal azul es como café con un cordón blanco...” (E2, 58),

tolerantes y que minimizan o normalizan las situaciones de abuso en general:

"yo creo que quedarme callada o sea, yo creo que eso hace que se mantiene, si tú no lo hacis notar, ellas están cómodas, te están torturando cómodamente..." $(E 7,34)$,

En relación a la forma de desarrollar su trabajo, también describen dos subgrupos. Las primeras lo ejecutan activamente y son descritas como personas metódicas, trabajólicas, a las que les gusta que el trabajo se haga bien, que no toleran que no se cumplan los acuerdos previos y que además se llevan bien con el personal que tienen a cargo, destacan entre sus pares y que ascienden rápidamente en el escalafón profesional.

En el segundo grupo, las personas se caracterizan por no poner límites al trabajo asignado, obedientes, sumisas, condescendientes y mantienen siempre en una posición inferior a sus jefaturas. Además sienten temor ante la posibilidad de perder el trabajo.

\section{Ejecución del rol profesional de la enfermera}

La claridad en la ejecución del rol profesional aparece, a juicio de las entrevistadas, como un factor central y esencial en la ocurrencia y mantención del hostigamiento laboral y se relaciona con dos grandes ejes, el primero relacionado con la formación profesional universitaria y el segundo, con la imagen social que se tiene de la enfermera. 
Estos dos ejes, junto con los contextos organizacionales ya mencionados, son descritos como asociados directamente a la causalidad del hostigamiento laboral en enfermería.

Las entrevistadas le adjudican un rol fundamental en la ocurrencia de hostigamiento laboral a la formación universitaria, tanto en lo referido a la formación en aspectos específicos de la profesión como a la formación en valores y actitudes de la enfermera.

De la formación específica en áreas de competencia profesional, las enfermeras describen una situación de indefensión técnica y profesional y falta de preparación, lo que estaría dado por una formación instrumentalizada:

“... como enfermeras que ellas entiendan que están para el cuidado y no para manejar máquinas,...yo veo que en general las enfermeras en este momento tienen una mala preparación, están muy instrumentalizadas para especialidades y todo, pero no están formadas integralmente." (E4, 143),

falta de desarrollo de un pensamiento crítico:

“...te acostumbran desde el día que entrai a la carrera a seguir indicaciones médicas, a que el médico es el que piensa y tu tenís que hacer las cosas que él pensó" (E7, 60),

falta de habilidades para manejar situaciones de estrés y un quehacer profesional derivado del cumplimiento de indicaciones médicas.

Por otro lado, la formación en actitudes y valores estaría marcada por una actitud de servicio, tolerancia, estoicidad, sumisión y auto postergación en función del cumplimiento del trabajo:

“... estar siempre en una actitud de servir pa' otros y no se preocupan de lo que tienen que hacer, es una actitud de de servicio patológico..." (E2, 42)“"

... yo creo que mientras más sometida más invisible y más fácil pasarla a llevar" (E4, 136)

Ambos aspectos influirían directamente en lo que ellas describen como falta de claridad para ejecutar el rol profesional lo que se vería reflejado en la inseguridad para desempeñar su trabajo:

“... las enfermeras yo creo que tiene tan poca, no sé, seguridad de lo que saben y tienen tan poco claro lo que hacen..." (E7, 54),

la poca visibilización del mismo:

"... yo creo que la enfermera no se ha preocupado de demostrar lo capaz que es, lo que es, lo que hace y cual es su verdadero rol...” (E4, 129)

y la falta de espíritu de cuerpo que se haría evidente a través de prácticas individualistas, rígidas, duras, fiscalizadoras y controladoras: 
“... yo creo que a las enfermeras nos encanta tener todo bajo control, mientras más llaves tenemos más felices somos, si tenemos más cosas a cargo, más felices somos..." (E4, 126).

En relación a la imagen social de la enfermera, las entrevistadas la describen sustentada en el cumplimiento de dos roles que determinan la valoración social de la profesión. El primero derivado del rol servicio, que en algunas ocasiones, incluso se confunde con la labor ejecutada por las religiosas y en que se destaca un trabajo caritativo, vocacional:

“...yo creo que aquí en este país... me da la idea que la opinión pública es de ser monja, virgen y Padre Hurtado, que está para servir, para agachar la cabeza, para hacer todo..."(E4, 127), abnegado ..."

O sea servicio, esta es una carrera de servicio, de abnegación, de que tú no estas acá pa' ganar plata, tú estás acá porque quieres servir a las otras personas...no podís exigir nada en enfermería"(E7, 63),

donde se debe cumplir con el mandato de "ser buena" y de servir permanentemente:

“... yo creo que por ahí va el cuento, porque tenis que ser buena, no podis ser mala...con mucha, mucha entrega... (E6, 65, 73).

El segundo rol está asociado a un rol técnico, supeditado al rol médico, instrumentalizado, sin autonomía ni independencia, que en algunos casos ni siquiera es considerado dentro de las profesiones de formación universitaria:

“...o sea la enfermera es una persona que está para entregar cuidados básicos...pero no se sabe que más hacen ¿una carrera universitaria?, no te lo puedo creer ¿y qué estudian?... (E7, 66).

De la respuesta a estos roles, según ellas, se desprendería la valoración social que se haría de la enfermera y que estaría determinada por un lado, por el nivel socioeconómico de la población, donde aparentemente, la enfermera sería mejor valorada y respetada en los niveles más bajos y desvirtuada en los más acomodados:

"... depende del estrato social, porque hay un estrato... bajo en que la enfermera está muy bien conceptuada, pero un estrato alto... en que se ha desvirtuado mucho la imagen porque en realidad pa' todo llaman a la enfermera...porque en los niveles más bajos tú eres dueña y señora y eres respetada..." (E8, 141)

y por el otro lado, por la valoración social asociada a la especialización dentro de la profesión, lo que se vería reflejado en las remuneraciones y en el reconocimiento social de la misma.

\section{Factores detonantes del comienzo del ciclo de hostigamiento}

Las enfermeras reconocieron situaciones específicas que provocaron un cambio en la relación que tenían hasta ese momento con la persona que luego actuó como hostigadora. En estos incidentes, además, es posible reconocer que el factor detonante se vincula con elementos de la relación existente entre ambas personas y 
no con factores externos, del contexto o el ambiente. Entre ellos describen que las personas que se transformaron en hostigadoras se sintieron amenazadas personal 0 profesionalmente por las víctimas:

“...ella estaba postulando a un trabajo y alguien a ella le comentó que yo había dicho que no quedó por la entrevista psicológica...” $(E 8,18)$

o bien, la víctima mostró actitudes asociadas a defender sus espacios profesionales y personales:

“... mandé un documento diciendo que no correspondía... que iba a revisar todo el instrumental, que iba a informar lo que no estaba bien..., pero que no me correspondía a mi asumir el costo de eso, que en el fondo na' que ver, osea bueno eso le cayó bomba... eso fue lo peor que pude haber hecho..." $(E 5,15)$.

\section{Conductas de hostigamiento laboral en enfermería}

En relación a las conductas de hostigamiento, las participantes concuerdan que éstas eran ejecutadas de manera solapada y manteniendo a las demás jefaturas sin conocimiento de los hechos:

“...entonces se te atan las manos, se te prohíben cosas, pero todo eso en forma verbal, nada por escrito, entonces cuando tú dices es que a mí me prohibieron hacer tal cosa, la directora dice "no, yo no he dicho nada"..." (E13, 20);

“...era como, hay es como si tú sintieras que están hablando de ti, que están tratando de perjudicarte, pero tú no ves a nadie...” (E5, 17),

y que se mantenían en el tiempo:

“... porque en la mañana llegan y te saludan con un beso y tú ves todo el día cómo te están poniendo trampitas y cortapisas y abriendo hoyitos en el suelo pa' que tú te caigas." (E13, 23).

Estas conductas ellas las dividen en aquellas que las afectan directamente en el ámbito personal y las que las afectan en el ámbito profesional.

En el ámbito personal describen conductas relacionadas con el trato que recibía la víctima, entre las que destaca, el castigo verbal, el trato en base a gritos, los empujones y las amenazas

“...miedo, me llegué a imaginar una vez que yo iba a salir de la clínica y ella me iba a estar esperando allí para hacerme algo, a ese, a ese nivel."(E9, 43);

rumores en torno a su persona, ridiculizaciones, burlas y otras conductas como cortarle el acceso telefónico, negarle vacaciones o restringirle bonos en dinero.

En el ámbito laboral se describen conductas ejecutadas con el fin de dañar a la víctima y que se pueden subdividir en dos categorías: 
- relacionadas con la ejecución del trabajo: en este grupo se describen comportamientos tales como el ocultamiento de información relevante, sobrecarga de trabajo “...dejándote el trabajo a ti, osea toda la agenda mía más la agenda de ella, osea, a mi me sobrecargaban de trabajo" (E13, 21), asignación de tareas inalcanzables o restricción de las responsabilidades de acuerdo a su cargo. Además, describen limitaciones para ocupar espacios físicos en los lugares de trabajo, cambios aleatorios de turnos y restricción de horas extraordinarias.

- relacionadas con el estatus profesional: en este grupo se describen conductas tales como acusaciones que les adjudicaban errores cometidos por otras personas, tergiversación de los situaciones relativas a decisiones laborales: "...que por eso yo había decidido cambiarme a turno, porque así tenía días disponibles... o sea le vendió a todo el mundo el cuento que había sido determinación mía y que yo lo había pedido y de que yo más encima estaba súper contenta con el cambio" (E10,30), descalificaciones en público “... tú aquí no eres nadie, tú no decides cosas, yo me voy a preocupar personalmente que te echen a patadas de aquí..." $(E 4,46)$, acusaciones sobre maltrato al personal: "...fue una situación en la que ella decía que yo le tenía mala y que yo siempre la criticaba y la maltrataba... habitualmente inventaba situaciones que no ocurrieron nunca y que ella se las hacía saber al resto del personal..." (E9, 22, 23), restricciones en los procesos de capacitación profesional "...no me mandaron más a capacitación, yo hace ya, van a hacer 3 años que no me mandan a ninguna capacitación..." (E13, 70), reclamos a las Direcciones de Servicio y bajas calificaciones de desempeño: “... por ejemplo, si tengo algún problema, no se po', si le contesté mal ella después me pone calificaciones malas o problemas con relaciones personales, ella maneja con eso, con el poder de las calificaciones..." $(E 3,41)$.

\section{Consecuencias que tuvo la experiencia de hostigamiento laboral en las enfermeras.}

Las enfermeras pudieron describir las consecuencias que sus experiencias de hostigamiento laboral produjeron en distintos ámbitos de su vida y que ellas definen como "negativas" específicamente en su salud y su trabajo.

En lo relativo a su salud, las enfermeras destacan consecuencias en su salud física y psicológica. Entre las primeras, la ocurrencia de cuadros como síndrome de intestino irritable, cervicalgias, presión precordial, temblores, palpitaciones, insomnio, pesadillas, baja de peso y vértigo. En el ámbito de su salud mental rescatan el dolor que esta situación les generó, muchas veces por lo inesperado del fenómeno, otras veces por tener la sensación de no merecer lo que les estaba ocurriendo, lo que se reflejó en sintomatología de la esfera ansiosa como por ejemplo, angustia, ansiedad, labilidad emocional; sintomatología de la esfera depresiva como por ejemplo, desilusión, ensimismamiento, aislamiento y tristeza:

"¿Y qué consecuencias tuvo para ti esto?... Devastadoras para mi, devastadoras desde el punto de vista psicológico... yo caí en una depresión, terminé en el psiquiatra..." $(E 13,60)$

y otros sentimientos como, por ejemplo, rabia, miedo, sensación de amenaza y agresión permanentes, de inseguridad, de indefensión y de traición, daños en la autoimagen y dudas acerca de si mismas y de su estabilidad mental: 
“...ella me transformó, era una hormiga, un gusano, una basura... era vieja y penca y me sentía aplanada...” (E11, 50),

"el que está sufriendo no sabe lo que está sufriendo, cree que es algo personal, que es algo imaginado y se viene a dar cuenta cuando la cosa está como en el desenlace" (E5, 124).

Además, destacan que estas consecuencias tuvieron repercusiones en su familia y otras relaciones sociales ya que proyectaban en ellos su agresión e irritabilidad:

"...porque yo andaba mal, yo andaba mal, entonces como que aaah... me trataba de desquitar así como que con todo el mundo..." (E8, 92).

En relación a su trabajo, las enfermeras describieron consecuencias en aspectos que dificultaron la relación con sus equipos de trabajos, lo que se manifestó a través de la desestabilización del personal o de actitudes descritas como de boicot del mismo hacia ellas, porque no querían trabajar, no cumplían sus órdenes o no seguían sus indicaciones. Sin embargo las consecuencias que describen como de mayor gravedad se relacionan con la pérdida de trabajo, ya sea porque decidieron dejar la institución, porque las despidieron o por último, porque las cambiaron aleatoriamente con evidentes repercusiones en los ingresos económicas de las mismas:

"...como consecuencia aparte de no subirme el sueldo me sacaron de ese puesto y me pusieron en turnos... o sea, lejos lo que yo menos quería era volver a hacer turnos..." (E10, 26).

\section{Estrategias de afrontamiento que utilizaron las víctimas para enfrentar las conductas de hostigamiento laboral.}

A partir de las descripciones que hicieron las enfermeras es posible describir dos formas de enfrentar las experiencias de hostigamiento: aquellas que les sirvieron para enfrentar activamente la situación y aquellas que utilizaron para sobrellevarla pasivamente.

Entre las primeras destacan cuatro tipos de conductas: las caracterizadas por la confrontación de las situaciones con los involucrados: “...yo me reúno con mis otras dos auxiliares, o sea las cuatro, y les digo a ver, está pasando esto, y ahí las versiones se entrecruzaron y nos dimos cuenta de lo que estaba pasando" (E 9, 34); las que recurren a instancias jerárquicas superiores para denunciar la situación y pedir ayuda; las relacionadas con activar redes intralaborales que les permitieran contrastar la situación con lo que otras personas observaban y por último, aquellas en que se recurrió a instancias legales y respaldo de documentos comprometedores. Además, en este grupo de enfermeras se destaca la elaboración cognitiva de los hechos que les permitieron analizar la situación específica y aclarar su posición frente a ella:

“... y cuando yo me di cuenta que no tenía ningún poder, ya no tuvo ningún poder sobre mí...” (E7, 70).

Por otro lado, entre los mecanismos que les servían para vivir pasivamente las situaciones de hostigamiento destacaron conductas relacionadas con no aclarar los 
acontecimientos ni pedir ayuda a sus pares o a instancias superiores por sentirse incapacitadas para ello, por lo que optaron por evitar el contacto con las personas involucradas concentrándose estrictamente en el desarrollo del trabajo asignado: "...yo diría que guardé silencio y traté casi de pasar inadvertida, osea casi de anularme y hacerme a un lado." (E12, 54), desarrollando actividades fuera del lugar de trabajo que les servían de distracción o haciendo usos de licencias médicas que justificaban la ausencia laboral: “...me fui con licencia... y yo empecé a dejar de trabajar, licencia, después de eso volvía a trabajar, licencia, volvía a trabajar, licencia... así estuve de julio a septiembre..." (E5, 66) y por último apoyándose en sus creencias religiosas

El uso de estas estrategias se relacionaba además con las motivaciones que las hacía mantenerse en el lugar de trabajo, entre ellas, la esperanza que la situación se revirtiera debido a un cambio de jefaturas, los vínculos afectivos que ahí habían desarrollado con otras personas, la comodidad que le generaban condiciones laborales como el horario y la inamovilidad del sistema público, el convencimiento que no encontrarían trabajo en otra parte: “... me infravaloré, yo pensé que nunca iba a encontrar trabajo y no iba a ser capaz de llegar a ningún otro lugar, porque me tenía tan, tan aplanada" $(E 11,47)$ o la dificultad que les generó darse cuenta de la situación por la que estaban pasando: “... bueno además el tiempo en que yo me di cuenta de todo este... porque me costó darme cuenta también po'... y cuando me di cuenta... ahí tomé la decisión" (E1, 28).

\section{Reacciones de los testigos frente al hostigamiento laboral de las víctimas.}

Las enfermeras describieron que los testigos (compañeros de trabajo y familiares) sólo se involucraron en la medida que ellas denunciaron los hechos y sus intervenciones consistieron en darles consejos, minimizar la situación o instándolas a agredir a las personas involucradas para responder a los ataques de los que eran objeto:

“...les cuento y me dicen, que sé yo, me dan consejos... embárrala de alguna forma... así como ella hace contigo, hazle tu también lo mismo..." (E3, 78)

o sugiriéndoles que buscaran otro trabajo.

Otra forma de intervención se relacionó con conductas concretas de contención emocional en las que destacaron la escucha y apoyo que las involucradas percibieron de quienes las ayudaban:

“... mis compañeras, las que sabían, empezaron como a solidarizar conmigo... o sea me acompañaron, me apoyaron, osea yo sentí que ellas fueron en ese momento pa' mi el apoyo pa' poder seguir en esa pega..." (E10, 43)

Sin embargo, también se hizo evidente que a pesar de saberlo, los testigos o se mantuvieron al margen de la situación, o a muchas de ellas no les creyeron lo que les estaba sucediendo:

“... mi marido nunca me agarró fíjate, no me pescó nunca, creía que eran cosas mías, que yo veía las cosas distorsionadas" (E11, 62, 63). 


\section{Definición de la experiencia.}

Las enfermeras definen sus experiencias de hostigamiento laboral de forma conceptual y metafórica. Los conceptos que utilizaron para definir conceptualmente la vivencia se ajustaron perfectamente al fenómeno. Entre ellos, abuso de poder, hostigamiento laboral, hostigamiento psicológico, acoso laboral, acoso psicológico y violencia laboral. Además destacan que estas situaciones son constantes, permanentes y mantenidas en el tiempo:

“... porque hostigamiento me suena a una cosa constante, osea que no es una vez y pasa sino que es algo que permanece, que se mantiene en el tiempo, osea el hecho de hostigar pa' mi es algo como que constantemente está ahí como, como punzándote..." (E10, 87).

Desde la definición metafórica de la situación se revela ampliamente el sufrimiento que la misma provocó en ellas “... en el fondo era mi alma la que lloraba” (E5, 66), lo inesperado de la situación "... te preparas para tomar algo dulce, te tomas tu tiempo, te preparas y te topas con un trago de hormigas" (E6, 141), la falta de recursos para afrontarla "...estaba cayendo en un hoyo y no tenía de donde agarrarme, me caía y me caía" $(E 7,42)$ y la necesidad de defenderse de ella: "... voy a la guerra, hay que usar camuflaje para defenderse”(E3,54), “... entonces uno tiene que empezar a caminar como en el medio de la jungla, mirando para todas partes porque tú sabes que los puñales pueden venir de cualquier lado" (E13, 23).

\section{DISCUSIÓN}

Las enfermeras participantes identifican como factores facilitadores y mantenedores del hostigamiento laboral a las características de la organización, la forma en que las enfermeras desarrollan su rol profesional, las características personales y profesionales de los actores involucrados, las reacciones de los testigos, la duración del fenómeno y las consecuencias en la vida de las víctimas.

En relación a los factores organizacionales, se destaca la ausencia de una política de resolución de conflictos, sistemas perversos de incentivos, los ambientes laborales y la forma en que actúan las jefaturas no directamente involucradas en los hechos. Estos hallazgos concuerdan con otros estudios que relacionan el soporte organizacional y clima organizacional con el desarrollo del trabajo de manera competente ${ }^{(13,14)}$, las actitudes y comportamientos de los jefes directos con la satisfacción laboral y la salud mental de las enfermeras ${ }^{(6)}$ y los ambientes laborales

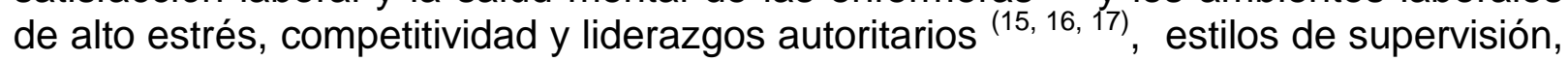
ausencia de interés y falta de apoyo por parte de los superiores y el tipo de relación del jefe con subordinados y/o compañeros ${ }^{(4,18,19)}$ con el hostigamiento laboral.

En relación a la imagen social y el desarrollo del rol profesional de la enfermera no es posible desconocer factores históricos relacionados con el desarrollo de la profesión que juegan un rol fundamental en la construcción de la imagen social de las mismas y en las características de la formación profesional recibida, ambos factores que han sido descritos como relevantes por las entrevistadas, para determinar la forma en que las enfermeras desarrollan y ejecutan su rol profesional.

De la imagen social aún es posible identificar elementos ligados a la herencia religiosa de la época de la conquista española y de la que se desprende la visión del rol 
profesional asociado a la tradición de servir y a la humildad (20), y por otro lado, elementos ligados al desarrollo de un rol técnico supeditado a la práctica médica que caracterizó a la época del Reformismo ${ }^{(21,22,23)}$. Sin duda, el fuerte desarrollo científico y disciplinar de la profesión de los últimos cincuenta años, no ha podido traspasar la barrera cultural, permeando al imaginario social y que según las mismas enfermeras, aún las posiciona primariamente en un rol de servicio no profesional que sería en parte reforzado por una formación universitaria que estaría muy influida por la sobrevaloración de la técnica y la dificultad para desarrollar actitudes que les permitieran posicionarse profesionalmente en el ejercicio del rol, entendiéndose éste como un rol ejecutado activa y autónomamente a partir de la reflexión crítica de las situaciones de enfermería.

Las enfermeras son capaces de distinguir las características que las hacen más susceptibles de ser hostigadas o de ejercer como hostigadoras, las que pudieran responder a características constitutivas de las mismas o al modelaje de la persona durante la formación, ya que debe considerarse que los modelos profesionales que intervienen en su proceso educativo, participan activamente en la construcción de su identidad como enfermeras y hacia la profesión ${ }^{(14,24)}$. Por otro lado, las enfermeras participantes y la evidencia disponible, describen que durante el proceso de formación muchas veces los alumnos son expuestos a hostigamiento por parte de los profesionales y docentes que participan en él, lo que se podría traducir en la incorporación de una pauta violenta de relación como modo habitual de desarrollar el trabajo, y que de alguna manera, invisibilizaría el fenómeno por considerarlo como constitutivo de la profesión, lo que se relaciona con los hallazgos de otros estudios, al referirse a cierto grado de aceptación de las situaciones violentas en los equipos de enfermería ${ }^{(6,25)}$.

Dentro de las conductas que en este grupo aparecen con mayor frecuencia, se encuentran el hostigamiento verbal y el ocultamiento de información relevante para el desarrollo del trabajo, lo que concuerda con los hallazgos de otros investigadores ${ }^{(1,4 \text {, }}$ $25,26,27)$, sumado a comportamientos tendientes a atacar la imagen pública de la víctima mediante la divulgación de rumores, amenazas al estatus profesional de la misma a través de acusaciones públicas sobre el desempeño en su trabajo, modificaciones de las características del trabajo asignado por debajo de las posibilidades de la víctima o dificultando la realización de las tareas asignadas, todos las cuales concuerdan con los resultados de otras investigaciones ${ }^{(4,28,29)}$.

\section{CONCLUSIÓN}

Los resultados obtenidos en esta investigación sumados a la evidencia internacional hacen innegable la necesidad de ampliar y profundizar el estudio del hostigamiento laboral en enfermería para dar cuenta de las características de este fenómeno en el contexto chileno y de la misma manera, la necesidad de profundizar en las características de la formación universitaria y del modelaje que reciben los estudiantes y que podría estar relacionado con el proceso de construcción de la identidad profesional y la mantención de valores culturales asociados al género y al ejercicio del poder.

En el ámbito laboral se hace necesario investigar aspectos relativos a la invisibilización de la violencia en los equipos de enfermería de forma tal que el hostigamiento laboral sea considerado como un problema que atenta contra el 
principio ético del respeto hacia las personas y sea definido como una situación inaceptable en las relaciones que se establecen al interior de los equipos de trabajo, independiente del rango y situación de los involucrados o de las características especificas de los contextos laborales.

La enfermería como profesión está a cargo de la gestión del cuidado de las personas, sin embargo, para que ello sea posible las enfermeras deben hacerse cargo de su propio cuidado y considerarse a sí mismas dignas de respeto y de desempeñar su trabajo en ambientes libres de violencia del cualquier tipo, partiendo indiscutiblemente por el buen trato entre los integrantes del equipo de enfermería.

De esta forma, la información sumada al trabajo preventivo y educativo se constituye como un imperativo moral del que no es posible hacer caso omiso si es que se quiere detener el flagelo del acoso moral en enfermería.

\section{REFERENCIAS BIBLIOGRÁFÍCAS}

1. Mayhew C, Chappell D. Workplace Violence in the Health Sector: A Case Study in Australia. The Journal of Occupational Health and Safety [internet]. 2003 [Consultado el 18 de noviembre de 2011]; 19(6). Disponible en http://www.who.int/violence injury prevention/violence/en/wpv australia.pdf

2. Amnistía Internacional. El Cuidado de los Derechos humanos: Oportunidades y desafíos para el personal de enfermería y partería. Madrid: Autor, 2006.

3. Kwok R, Law Y, Li K, Ng Y, Cheung M, Fung V, et al. Prevalence of workplace violence against nurses in Hong Kong. Hong Kong Med J. [internet]. 2006 [Consultado el 20 de noviembre de 2011]; 12(1): 6-9. Disponible en http://www.hkmj.org/article pdfs/hkm0602p6.pdf.

4. Johnson SL. International perspectives on workplace bullying among nurses: a review. International Nursing Review [internet]. 2009 [Consultado el 23 de noviembre de 2011]; 56: 34-40. Disponible en http://onlinelibrary.wiley.com.ezproxy.puc.cl/doi/10.1111/j.14667657.2008.00679.x/pdf

5. Efe SY, Ayaz S. Mobbing against nurses in the workplace in Turkey. International Nursing Review [internet].2010 [Consultado el 20 de noviembre de 2011]; 57: 328-34. Disponible en http://onlinelibrary.wiley.com.ezproxy.puc.cl/doi/10.1111/j.14667657.2010.00815.x/pdf

6. Royal College of Nursing. (2002). Working well: a call to employers [internet]. 2002 [Consultado el 5 de diciembre de 2011]. Disponible en http://www.rcn.org.uk/members/downloads/working well summary.pdf

7. Charmaz, K. Grounded theory in the $21^{\text {st }}$ century: Applications for social justice studies. En N. Denzin \& Y. Lincoln (Eds.), Handbook of qualitative research $\left(3^{a}\right.$ ed., pp. 507-535). Thousand Oaks, CA: Sage, 2005.

8. Dantas CC, Leite JL, Lima SBS, Stipp MAC. Teoría fundamentada en los datos - aspectos conceptuales y operacionales: metodología posible de ser aplicada en la investigación en enfermería. Rev. Latino-am. Enfermagem [internet]. 2009 [Consultado el 15 junio 2011]; 17(4): 573-579. Disponible en http://www.scielo.br/scielo.php?script=sci_arttext\&pid=S0104$11692009000400021 \&$ lng=pt.

9. Sandoval C. Investigación Cualitativa. Colombia: ARFO, 1996.

10. Flick, U. Introducción a la investigación cualitativa. Madrid: Morata, 2004. 
11.Strauss A, Corbin J. Basics of qualitative research. California, USA: Sage, 1990.

12. Guba, E. The alternative paradigma dialog. En E. Guba (Ed.), The paradigm dialog. Newbury Park: Sage, 1990.

13. Ying L, Kunaviktikul W, Tonmukayakal $O$. Nursing competency and organizational climate as perceived by staff nurses in a Chinese university hospital. Nurs Health Sci.2007; 9(3): 221-7.

14. Topa G, Moriano JA, Morales JF. Acoso laboral entre profesionales de enfermería: El papel protector de la identidad social en el trabajo. Anales de Psicología. 2009; 25(2): 266-76.

15. Einarsen S, Raknes BI, Matthiesen SM, Hellesoy OH. Bullyng and harassment at work and their relations to work environment quality. An exploratory study. The European Work and Organizational Psychology. 1994; 4: 381-401.

16. Einarsen S, Raknes BI, Matthiesen SM, Hellesoy $\mathrm{OH}$. Bullying and harsh personalized conflicts: Unhealthy interaction at work. Bergen, Norway: Sigma Forlag, 1994.

17. Moreno B, Rodríguez A, Garrosa E, Morante ME. Acoso psicológico en el trabajo; una aproximación organizacional. Revista de Psicología del Trabajo y de las Organizaciones, 2004; 20(3):277-89.

18. Moreno B, Rodríguez A, Garrosa E, Morante ME. Antecedentes organizacionales del acoso psicológico en el trabajo: Un estudio exploratorio. Psicothema. 2005; 17(4): 627-32.

19. Luna M. Acoso Psicológico en el Trabajo (Mobbing). Secretaría de Salud Laboral de CC.OO. Madrid: GPS, 2003.

20.Fajardo ME, Germán C. Influencia del género en el reconocimiento de los cuidados enfermeros visibles e invisibles. Index Enferm. 2004; 13(46):09-12.

21. Hernández J. Historia de la Enfermería: Un análisis histórico de los cuidados de enfermería. Madrid: Mc Graw Hill, 1995.

22. Lillo M. El Desarrollo de la Enfermería Moderna. El Reformismo Británico del siglo XIX como agente político de cambio. Index de Enferm.[internet]. 2002 [Consultado el 5 de noviembre de 2011]; 36-37:33-36. Disponible en http://www.index-f.com/index-enfermeria/36-37revista/36-37 articulo 33-36.php $\underline{3}$

23. Velandia A. (2005). Influencias étnicas en la Enfermería latinoamericana. Temperamentvm 1.[internet]. 2005 [Consultado el 5 de noviembre de 2011]. Disponible en http://www.index-f.com/temperamentum/1 revista/a0101.php

24. Loo I. La identidad como proceso biológico-psicosocial y su construcción en enfermería. Revista Enfermería. 2003; 11(1): 49-54.

25.Thomas, S.P., \& Burk, R. Junior nursing students' experiences of vertical violence during clinical rotations. Nursing Outlook. 2009; 57: 226-31.

26. Atawneh F, Zahid M, Al-Sahlawi K, Shahid A, Al-Farrah M. Violence against nurses in hospitals: Prevalence and effects. BJN. 2003: 12:102-7.

27. Hoel H, Cooper C. Destructive conflict and Bullying at work [internet]. Manchester School of Management, Institute of Science and Technology, University of Manchester; 2000. Disponible en http://www.rowleyassoc.com/wpcontent/uploads/Conflict-Bullying-at-Work.pdf

28. Rayner C, Hoel H. A summary review of literature relating to workplace bullying. Journal of Community and Applied Social Psychology.[internet].1997 [Consultado el 26 de noviembre de 2011]; 7 (3):181-91. Disponible en http://www3.interscience.wiley.com/cgibin/accessdenied?ID=14384\&Act $=2138 \&$ Code $=4717 \&$ Page $=/$ cgibin/fulltext/14384/PDFSTART 
29. Fornés J, Martínez MA, García G. Análisis factorial del cuestionario de hostigamiento psicológico en el trabajo en profesionales de enfermería. Int $\mathrm{J}$ Clin Health Psychol [internet]. 2008 [Consultado el 5 de enero de 2012]; 8(1):267-83. Disponible en http://redalyc.uaemex.mx/pdf/337/33780118.pdf 